\title{
2250-MHz High Efficiency Microwave Power Amplifier (HEMPA)
}

\author{
Dr. W. Herbert Sims
}

\author{
Radio Frequency Group, Avionics Department of the Engineering Directorate, Marshall Space Flight Center, \\ Huntsville, AL 35812; (256) 544-8581, herb.sims@msfc.nasa.gov
}

\begin{abstract}
This paper will focus on developing an exotic switching technique that enhances the DC-to-RF conversion efficiency of microwave power amplifiers. For years, switching techniques implemented in the $10 \mathrm{kHz}$ to $30 \mathrm{MHz}$ region have resulted in DC-to-RF conversion efficiencies of 90-95-percent. Currently amplifier conversion efficiency, in the $2-3$ $\mathrm{GHz}$ region approaches, 10-20-percent. Using a combination of analytical modeling and hardware testing, a High Efficiency Microwave Power Amplifier was built that demonstrated conversion efficiencies four to five times higher than current state of the art.
\end{abstract}

\section{INTRODUCTION}

Since the Space Age began, various microwave power amplifier designs that used Class-A, $-\mathrm{B}$, and $-\mathrm{C}$ bias arrangements have been employed. However, a common limitation of these amplifiers is the high input power required to generate the RF/microwave power. In fact, the microwave amplifier has typically been the largest drain for the limited power available on the spacecraft because its conversion efficiency is only 10- to 20-percent. In contrast, state of the art (SOA) Low-Frequency (LF) to High Frequency (HF) amplifiers commonly attain a conversion efficiency of 80-95-percent. To achieve high efficiency in that region of the RF spectrum, a Class-D bias configuration must be used. Therefore, it seemed reasonable that a Class-D bias configuration might also produce high efficiencies in the microwave frequency band.

Baxandall (Raab, 1982) first designated the Class-D terminology in the early 1960s while working with transistor sine wave generators from switching mode oscillators. The Class-D amplifier has several distinguishing characteristics:

- It has both high voltage across the device and large current through the device, though not simultaneously;

- It is composed of one or more transistors operating as single-pole switches; and

- The frequency of the output signal is the fundamental switching frequency.

Little research has addressed Class-D microwave amplifiers - called High-Efficiency Microwave Power Amplifiers (HEMPA). Therefore, a 2250-MHz HEMPA was designed and tested to determine whether the power conversion efficiency of SOA microwave amplifiers could be significantly raised. This case study involved parameter extraction and modeling of microwave power GaAs FET devices and then with assessing the appropriate power divider topology. Finally, a single-stage power amplifier was built and tested to determine its conversion efficiency and gain.

\section{ANALYTICAL MODELING}

In the past, many hours were spent in the "what-if" method of designing a new microwave power amplifier. Today, the use of high-speed computers condenses this "what-if" method so that multiple amplifier configurations can be accurately simulated in far less time than a single configuration took previously. The simulation of a HEMPA was implemented by taking three distinct steps. First, the transistors were modeled using the HP EEFet3 (Hewlett/Packard, 1981-2000) model incorporated in IC-CAP (Hewlett/Packard, 1981-2000) to extract particular transistor parameters. That information, in turn, was used in a jOmega (Hewlett/Packard, 1981- 
2000) simulation, which is a general-purpose RF/microwave circuit simulation program developed by HP/EESof for nonlinear DC, nonlinear transient and linear AC analyses. The HP EEFet 3 model is described in detail below.

\section{Microwave Transistor Models}

The HP EEFet 3 model is an empirical model developed for fitting measured electrical behavior of GaAs FETs. The model includes the following features:

- An accurate isothermal drain-source current model that fits virtually all processes:

- A self-heating correction for the drain-source current;

- A charge model that accurately emulates measured capacitance values;

- A dispersion model that permits simultaneous fitting of high-frequency conductances and DC characteristics;

- A breakdown model that describes gate-drain current as a function of both gateto-source and drain-to-source voltage, $\mathrm{V}_{\mathrm{gs}}$ and $\mathrm{V}_{\mathrm{ds}}$ respectively;

- The capability to extrapolate outside the measurement range used to extract the model.

The high-frequency FET modeling software IC-CAP performs a series of DC- and S-Parameter measurements, based on predefined measurement configurations and on variables defined during the procedures outlined below. The measured values are then used to extract individual device parameters through software conversion of the S-parameters to admittance or impedance parameters. The resulting model can then be used to simulate the performance of the actual device in a circuit. The procedures involved use a series of setpoints to measure current or voltage vs. bias under different bias conditions. Setpoints serve to decouple the model equations and to effectively isolate the individual FET parameters. The following ten procedures are used to isolate these parameters:

- Device preview - verifies proper device operation;

- Measure drain current as a function of swept gate voltage;

- Vary the drain current with respect to drain voltages, at several values of gate voltage (Family of Curves);

- Preview of source, drain and gate resistances using Yang-Long method;

- Final Yang-Long measurement;

- Extraction of intrinsic and extrinsic parasitics from S-parameter data;

- Measure Ideality, I, of FET;

- Measure the values of $\mathrm{V}_{g}$ with $\mathrm{V}_{\mathrm{ds}}$ at a constant value of $\mathrm{V}_{\mathrm{ds} 0}$;

- Measure $I_{d s}$ vs. $V_{d s}$ while varying $V_{g s}$; and

- Measure swept S-parameters.

Once a complete set of data is collected for a particular device, the extraction proceeds by iteratively determining the proper small-signal model parameter values. In the governing equations, the voltages are assumed intrinsic. The HP EEFet 3 model uses intrinsic voltages to predict device performance. However, since there is no known way to directly measure or to set the intrinsic voltages, they are calculated using the parasitic resistances and inductances and the known currents.

The converged solutions are reliable and accurate. Figure 1 compares the simulated and measured $S_{11}$ and $S_{22}$ parameters of the NEC-900 I-75 model. 


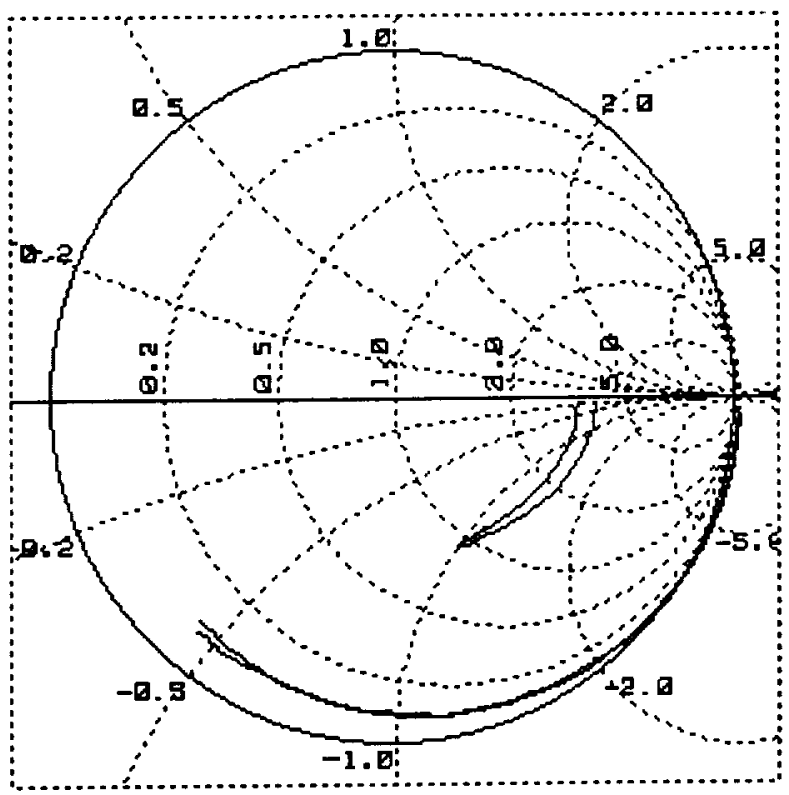

FIGURE 1. Actual vs. simulated $S_{11}$ and $S_{22}$ parameters for the NEC 9001-75 Device.

\section{HEMPA Model}

Most nonlinear circuit analysis programs that exist today were designed primarily for transient analysis. However, they are not often adequate when the designer needs to simulate GaAs FETs that are required to operate at high DC-to-RF conversion efficiencies -- a more sophisticated model is needed. One method that works well in this arena is called harmonic-balance, as described by Quéré et al. (1993).

The harmonic-balance method is iterative in nature and is based on the assumption that, for a given sinusoidal excitation, a steady state solution exists that can be approximated with a finite Fourier series. With the solution postulated in the form of a finite Fourier series, the circuit node voltages take on a set of amplitudes and phases for all frequency components. The currents flowing from nodes into linear elements, including all distributed elements, are calculated by a frequency domain linear analysis. Currents from nodes into nonlinear elements are calculated in the time domain. Generalized Fourier analysis is used to transform from the time-domain to the frequency-domain.

The jOmega simulation is the implementation of a harmonic-balance analysis.

\section{Power Divider for Harmonically Rich Waveforms ${ }^{(S i m s, 1997)}$}

A major obstacle to overcome in the design and development of a HEMPA dealt with the accurate division of a single harmonically rich square wave signal into two equal-amplitude, opposite-phase signals. Current divider topologies - like the Wilkinson hybrid, the radial wave power hybrid (Swift, 1988) and the multiport power divider using circular-sector-shaped planar components (Abouzahra, 1988) -- are not well suited for this application due to bandwidth constraints. Furthermore, Figure 2 illustrates another reason current divider topologies fail: the output frequencies are in-phase only at $\lambda / 2$ and $\lambda$. 


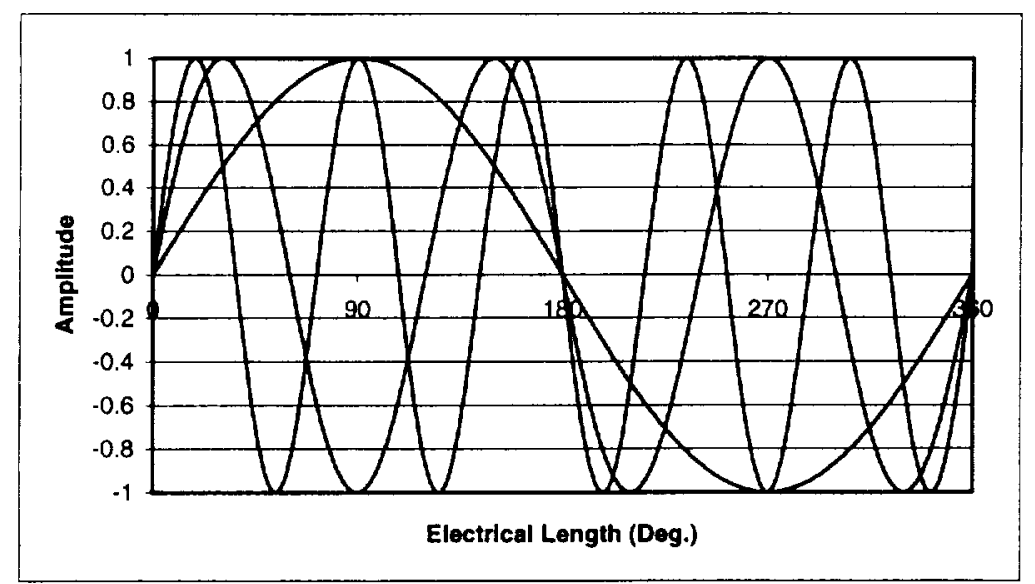

FIGURE 2. Normalized phase vs. frequency.

Therefore, the development of a new topology was required to propagate square wave signals more efficiently. This new topology uses a push-pull configuration in the amplifier, which results in greater power generating capability. Furthermore, unique requirements for a HEMPA drove two significant features:

- The fundamental frequency and all odd ordered harmonics must maintain their phase and amplitude relationship;

- Each frequency component, normalized to the fundamental, must be phase coherent at the output.

All simulations to verify the above theory used transmission lines in the jOmega model. The simulation used two transmission lines: one that was $\lambda / 2$ in total length and the other being $\lambda$ in total length, where the frequency of interest was $2 \mathrm{GHz}$. Initially, ideal transmission lines were assumed, but verification of theory was also carried out using a non-ideal microstrip, since the ideal does not exist in practice. The microstripline measurements generated in the simulation were used in the layout portion of jOmega, and the final board geometry, shown in Figure 3, is 2.0inches in length and 1.0-inches in width.

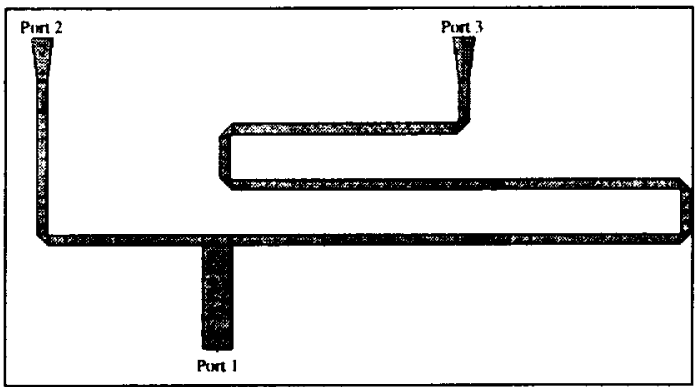

FIGURE 3. Power divider for harmonically rich waveforms.

\section{0-MHz HEMPA DESIGN AND TEST}

Following the simulations to validate the theory of a Class- $\mathrm{D}$ microwave amplifier, a single stage, $2.25-\mathrm{GHz}$ amplifier was built and tested, and is shown in Figure 4. There were multiple objectives of these tests.

- Validate IC-Cap Models;

- Validate power divider/combiner;

- Validate jOmega RF models;

- Efficiency of conversion of sine-wave to square-wave; 


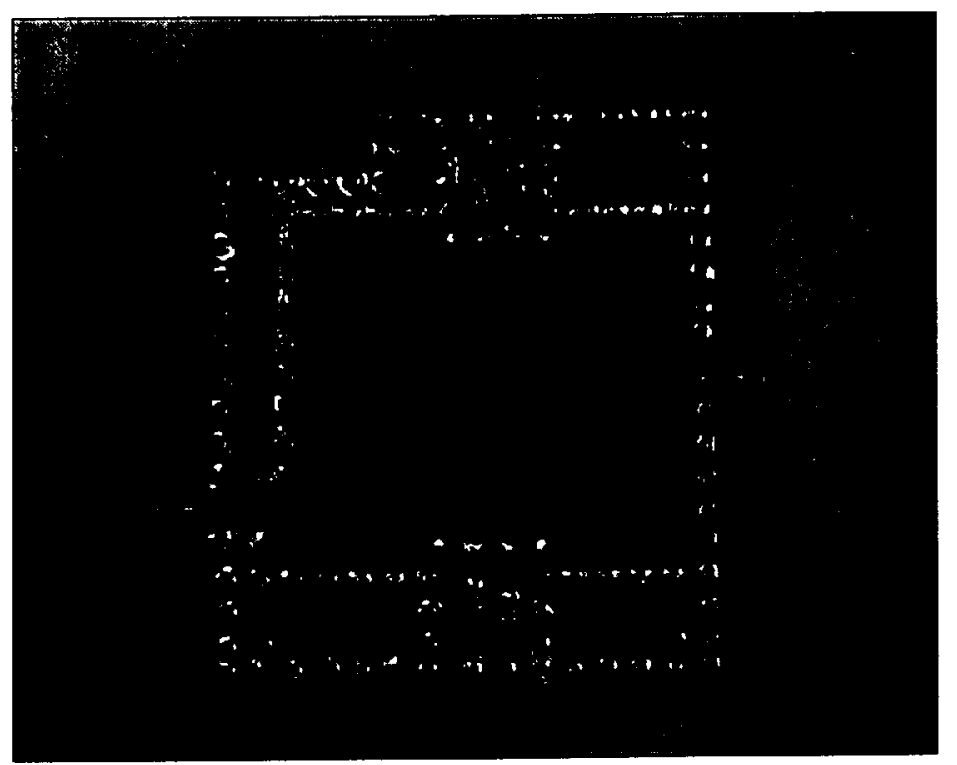

Figure 4. Single stage layout - high efficiency microwave power amplifier.

Similar to the power divider sub-circuit, the first topology simulated used ideal models- the only exception being the transistor models. As the analysis continued, the ideal components, including resistors, inductors and capacitors, were replaced with practical models. Again, the reason for this multi-step approach was to verify several essential issues specific to a HEMPA. The first issues deals with an efficient method of converting the sine wave receiver/exciter (R/E) signal to a square-wave. Once the waveform is converted, accurate amplification of the signal can proceed. The second issue deals with the improvement of the input VSWR. Because the amplifier is in either cut-off or saturation, the input impedance varies dramatically between these two extremes.

Using standard lab test equipment, performance measurements were taken. The objective of the first experiment was to vary the drain and gate, $\mathrm{V}_{\mathrm{ds}}$ and $\mathrm{V}_{\mathrm{gs}}$ respectively, voltages to find the biasing arrangement that resulted in maximum efficiency with maximum gain. $V_{\mathrm{gs}}$ was varied from -2.5 to $-1.0 \mathrm{VDC}$ in 0.1 -VDC increments and $\mathrm{V}_{\mathrm{ds}}$ was varied from 2.5 to $4.5 \mathrm{VDC}$ in 0.25 - VDC increments. The amplifier gain relative to the fundamental frequency $(2250 \mathrm{MHz})$ with input power of $+10-\mathrm{dBm}$ was also measured. Additionally the drain current, $I_{d s}$ in milliamps, was measured to determine the input $D C$ current. Using equations (1) and (2), along with the reflected power in milliwatts was determined.

$$
P_{\text {ref }}=P_{\text {in }} \rho
$$

Where $P_{\text {in }}$ is the incident power (in this case $+10-\mathrm{dBm}$ ) and $\rho$ is the reflection coefficient given by

$$
\rho=\frac{(V S W R-1)}{(V S W R+1)}
$$

The actual output power, from the gain and VSWR-corrected power was determined. Using equations (1) and (2), the efficiency vs. $\mathrm{V}_{\mathrm{ds}} / \mathrm{V}_{\mathrm{gs}}$ can be determined.

$$
\eta=\frac{P_{i u t}}{P_{D C}+\left(P_{i n}-P_{r e f}\right)}
$$

Because both the gain and efficiency of the amplifier are a function of $V_{\mathrm{ds}}, \mathrm{V}_{\mathrm{gs}}$, and $\mathrm{I}_{\mathrm{ds}}$, one can take the values of efficiency vs. $V_{d s} V_{g s}$ and multiply them by the gain values to generate a maximum gain-efficiency plot. This is shown in Figure 5. 


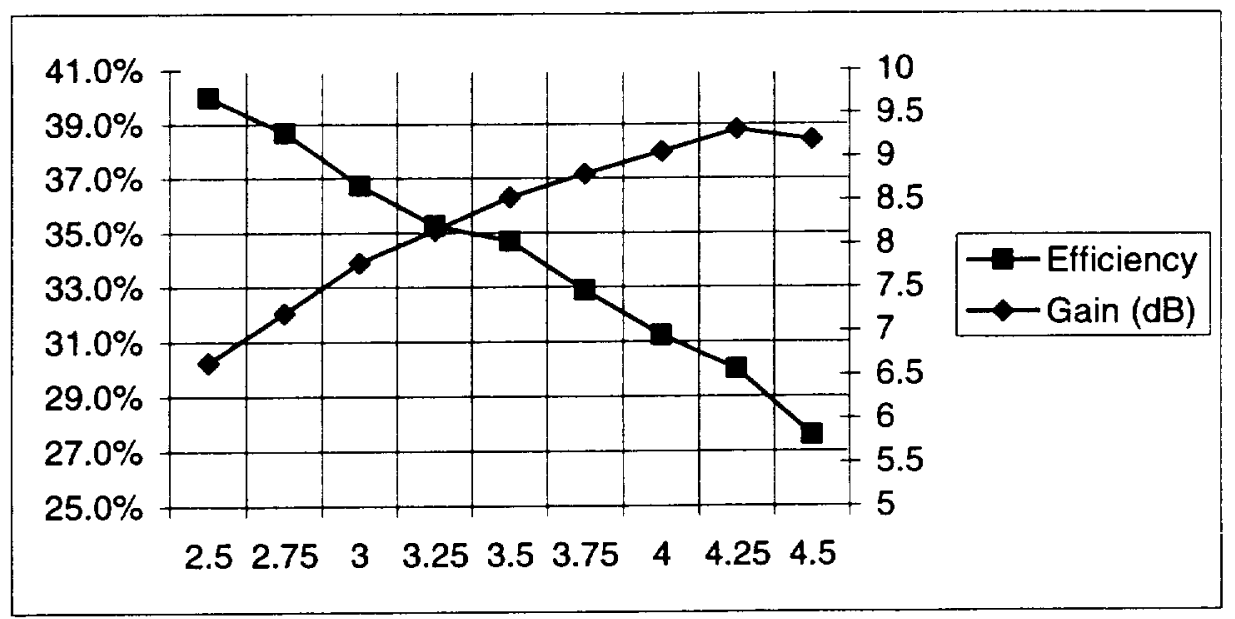

FIGURE 5. Gain and efficiency vs. $V_{\mathrm{ds}}$.

The result from this multiplication shows that for an approximate $10 \%$ decrease in the maximum efficiency (where $\mathrm{V}_{\mathrm{ds}}=2.5 \mathrm{VDC}$, and $\mathrm{V}_{\mathrm{gs}}=-3.0 \mathrm{VDC}$ ) an increase of 3-dB in gain (where $\mathrm{V}_{\mathrm{ds}}=3.75 \mathrm{VDC}$, and $\mathrm{V}_{\mathrm{gs}}=-2.0$ VDC) can be achieved.

The final amplifier exhibits 9.11-dB of gain with an efficiency of 40.2-percent - which is quadruple the efficiency of a typical S-band amplifier. If the efficiency is considered more important than the gain then maximum efficiency of the stage could be biased for 49.5-percent efficiency, but with a gain of only 6.72-dB.

\section{CONCLUSIONS}

This paper develops the premise that microwave amplifier efficiency can be increased from the standard 10percent to approximately 40-percent, a four-fold increase in efficiency. Proof of principle is demonstrated here through a specific $2250 \mathrm{MHz}$ case study.

\section{RECOMMENDATIONS}

Overall, for a new design of a high-efficiency microwave power amplifier, the outcomes were positive. In this paper, it was shown that, at a minimum, the overall efficiency of a typical microwave amplifier can be improved to approximately 40-percent. To further exploit these results, three recommendations for future work are offered.

The first recommendation is the need to increase the fidelity of the power device models. The IC-CAP software is helpful in extracting and generating the individual transistor parameters. However, since no system is completely accurate, better transistor models should be pursued. During this research, hundreds of iterations were required to generate a usable transistor model.

The second recommendation is to improve on the ability of jOmega to accurately complete a simulation. Special techniques had to be incorporated into the simulation-input file to generate meaningful results. Convergence errors needed special attention, arising from two unrelated simulation requirements. The first requirement is highpower level simulations. As the power levels involved with the circuit are increased, there is a threshold where jOmega becomes unstable. Second, special input file inclusions alleviated some of these problems, but each time a circuit parameter is employed, the technique has to be changed to allow a successful simulation.

The second simulation requirement is the need for bias-dependent models currently used. Currently, jOmega can only do non-linear simulations on bias-dependent devices. This is a severe limitation when trying to simulate Class-D amplifiers, where the device is in either cut-off or saturation. Some form of multiple bias levels in the model must be undertaken. 
The third recommendation is for more work on the advancement of wider bandwidth power devices. Currently, the devices being used have a unity gain at approximately $18 \mathrm{GHz}$. As this is increased through future revisions to the actual power devices, more efficient power amplifiers can be achieved. As the technology improves, so will the Class-D amplifier.

\section{SUMMARY}

This paper establishes HEMPAs as capable of operating in the microwave region to at least several GHz. For years, HEMPAs have been used in much lower frequency bands with great success-at 95 -99-percent efficiency. As the device technology improves, this research provider reasons to believe HEMPAs will see an increasing popularity at progressively higher frequencies in the future.

\section{REFERENCES}

Abouzahra, M.D., Gupta, K.C., "Multiport Power Divider - Combiner Circuit Using Circular-Sector Shaped Planar Components," IEEE Transactions on Microwave Theory and Techniques, Vol. 36, No. 12, December, 1988.

Hewlett/Packard-EESof, 5601 Lindero Canyon Road, Westlake Village, CA 91362, (818) 879-6200.

Quéré, R., NGoya, E., Teyssier, J.P., Rousset, J., Obregon, J., "Semiconductor Modeling for Harmonic Balance Simulation," SBMO International Microwave Conference, 1993.

Raab, F.H., "Analysis of Idealized Class-D Power Amplifiers," Green Mountain Radio Research Company Continuing Education Seminar, Vermont, 1982.

Sims, William H., III, "Power Divider for Harmonically Rich Waveforms," NASA Case No. MSF-31186-1, 1997.

Swift, G.W., Stones, D.I., “A Comprehensive Design Technique for the Radial Wave Power Combiner," IEEE Microwave Theory and Techniques, 1988. 